\title{
Comunicação direta entre artéria pulmonar direita e átrio esquerdo: relato de dois casos
}

\author{
Luiz Carlos SCHIMIN*; Itacir Arlindo FRANCESCHINI*; Jean Newton Lima COSTA*; \\ Vinícius Sousa de LUCENA*
}

RBCCV 44205-522

Schimin L C, Franceschini I A, Costa J N L, Lucena V S - Comunicação direta entre artéria pulmonar direita e átrio esquerdo: relato de dois casos. Rev Bras Cir Cardiovasc 2000; 15(4): 349-52.

RESUMO: A comunicação direta entre a artéria pulmonar direita e o átrio esquerdo é uma condição rara, tendo sido relatados menos de 40 casos em toda a literatura. Apresentação clínica é variável, podendo o paciente mostrar-se com quadro de cianose, dispnéia ou insuficiência cardíaca. No entanto, o diagnóstico pode ser facilmente feito com métodos de imagem. O tratamento cirúrgico oferece a cura completa com baixa morbi-mortalidade. Sem o mesmo, sérias complicações podem advir como embolia ou abscesso cerebral. Os autores apresentam dois casos de comunicação direta entre artéria pulmonar direita e átrio esquerdo. São discutidas as teorias embriogênicas e as variantes anatômicas desta anomalia congênita.

DESCRITORES: Procedimentos cirúrgicos cardíacos, métodos. Átrio cardíaco, cirurgia. Artéria pulmonar, cirurgia.

\section{INTRODUÇÃO}

Comunicação direta entre artéria pulmonar direita $(A P D)$ e átrio esquerdo $(A E)$ é uma cardiopatia congênita rara e tem sido reconhecida como uma categoria diagnóstica específica. Suas características semiológicas, terapêuticas e prognosticas a tornam uma entidade nosológica bem distinta das fístulas artério-venosas pulmonares ${ }^{(1)}$. O manuseio clínico isoladamente pode levar a complicações sérias e até à morte, sendo que a cura completa só pode ser obtida com o tratamento cirúrgico.

\section{RELATO DE CASOS}

\section{Caso № 1}

RN com 1 dia de vida, sexo masculino, branco com 3700 g. Apresentava cianose com gasometria evidenciando saturação extremamente baixa $\left(\mathrm{pO}_{2}=19 \mathrm{mmHg}\right.$, sat. $\left.\mathrm{O}_{2}=27 \%\right)$. O eletrocardiograma mostrava discreta sobrecarga ventricular esquerda (SVE). Radiografia de tórax normal. O exame angiográfico revelou comunicação direta artéria

\footnotetext{
Trabalho realizado no Hospital de Base do Distrito Federal (HBDF). Brasília, DF, Brasil.

Recebido para publicação em março de 2000.

* Do Hospital de Base de Brasília.

Endereço para correspondência: Luiz C. Schimin - SHIS QL 8 Cj. 6 Cs. 5. Brasília, DF, Brasil. CEP: 71620-265. Tel: (61) $248-0771$.

e-mail:schimin@tba.com.br
} 
Schimin L C, Franceschini I A, Costa J N L, Lucena V S - Comunicação direta entre artéria pulmonar direita e átrio esquerdo: relato de dois casos. Rev Bras Cir Cardiovasc 2000; 15(4): 349-52.

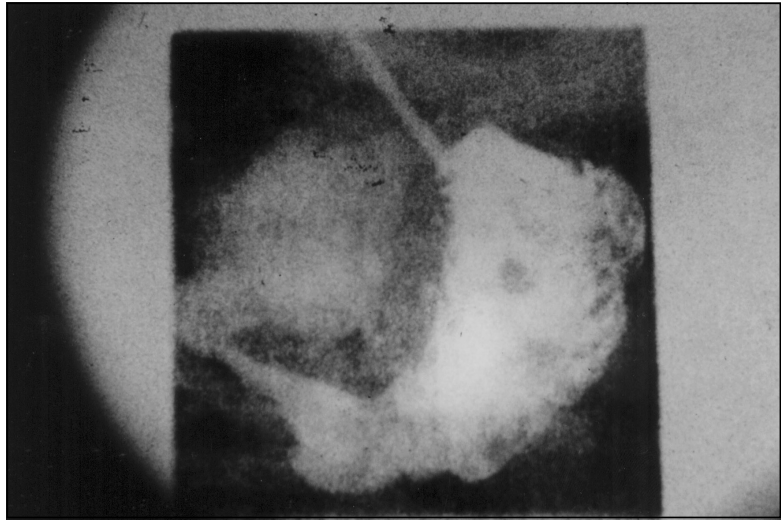

Fig. 1 - Angiografia - árido esquerdo evidenciado a partir de contraste injetado em artéria pulmonar

pulmonar - átrio esquerdo (Figura 1). A criança foi submetida a toracotomia esquerda e ligadura do vaso que nos pareceu anômalo, próximo ao lobo pulmonar inferior esquerdo (LIE). Evoluiu mal no período pós-operatório imediato, com importante cianose e dessaturação arterial de $\mathrm{O}_{2}$. A radiografia de tórax mostrava hiperinsuflação do pulmão direito. Realizada arteriografia pulmonar, encontramos ausência de circulação para a base pulmonar esquerda. O RN foi levado a nova operação por esternotomia mediana, desfeita a ligadura do vaso (ramo da artéria pulmonar esquerda para o LIE) e foi identificada estrutura anômala que comunicava a APD com AE. O vaso anômalo foi ligado, obtendo-se melhora imediata da sat. $\mathrm{O}_{2}(98,9 \%)$. A criança faleceu no 350 dia de vida, com atelectasia pulmonar e pneumonia.

\section{Caso № 2}

Criança de 5 anos, sexo feminino, branca Cianose desde o nascimento. Acompanhada periodicamente para esclarecimento diagnóstico. Aos 3 anos, esteve internada nos setores de Hematologia e Pneumologia, para investigação. Foi afastada hemoglobinopatia. A cintilografia pulmonar sugeria fístula artério-venosa (FAV) na circulação pulmonar. O ecocardiograma evidenciava discreto aumento de AE.

Foi admitida no setor de Cardiologia para avaliação e investigação da FAV pulmonar. Apresentava bom desenvolvimento. Eupnéica, cianótica $(+++/ 4)$ com baqueteamento digital. Ausculta cardiopulmonar normal. Radiografia de tórax e eletrocardiograma evidenciavam discreto aumento de VE.

O cateterismo cardíaco com arteriografia pulmonar mostrou opacificação precoce do $A E$ a partir do ramo direito da AP (Figura 2).

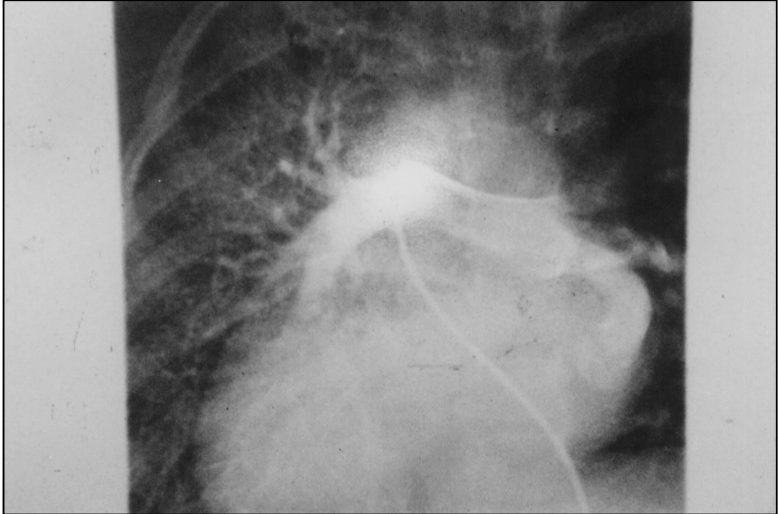

Fig. 2 - Angiografia mostrando vaso anômalo conectando ramo direito da artéria pulmonar e átrio esquerdo.

O paciente foi submetido a esternotomia mediana. Identificada estrutura anômala de aproximadamente $5 \mathrm{~mm}$ de diâmetro e $3 \mathrm{~cm}$ de comprimento que conectava APD-AE (Figura 3). O vaso anômalo foi dissecado. Nenhuma veia pulmonar drenava nele. Procedemos à ligadura do mesmo, havendo imediato aumento da saturação de $\mathrm{O}_{2}(95 \%)$. O procedimento foi realizado sem circulação extracorpórea (CEC). A criança recebeu alta médica no 7으 dia pósoperatório, em bom estado geral, acianótica, com sat. $\mathrm{O}_{2}=97 \%$.

\section{COMENTÁRIOS}

A comunicação direta da $A P$ ou seus ramos para o $A E$ é uma condição rara. São relatados 35 casos em toda a literatura (2). Os casos relatados têm idade que variam de 1 dia a 45 anos, porém apenas $6 \mathrm{com}$ menos de 1 ano(3-5).

Os sinais e sintomas mais freqüentes são dispnéia e cianose, sendo a insuficiência cardíaca mais rara ${ }^{(2,6)}$. Alguns pacientes apresentam sopros cardíacos, que são em grande parte inespecíficos (3). Pode, no entanto, ser evidenciado sopro contínuo descrito em região interescapulovertebral direita ${ }^{(7,8)}$. O eletrocardiograma é normal ou apresenta discreta sobrecarga de ventrículo direito, o que elimina grande parte das cardiopatias congênitas cianóticas. A radiografia de tórax pode mostrar massa para-hilar direita, orientando o diagnóstico para FAV pulmonar ${ }^{(9)}$. O cateterismo cardíaco com angiografia é fundamental para o diagnóstico e programação cirúrgica. A ressonância nuclear magnética tem possibilitado igualmente o diagnóstico desta anomalia (2), mas ainda tem uso bastante restrito.

A presente condição pode ser explicada por uma conexão precoce entre artéria pulmonar (AP) 


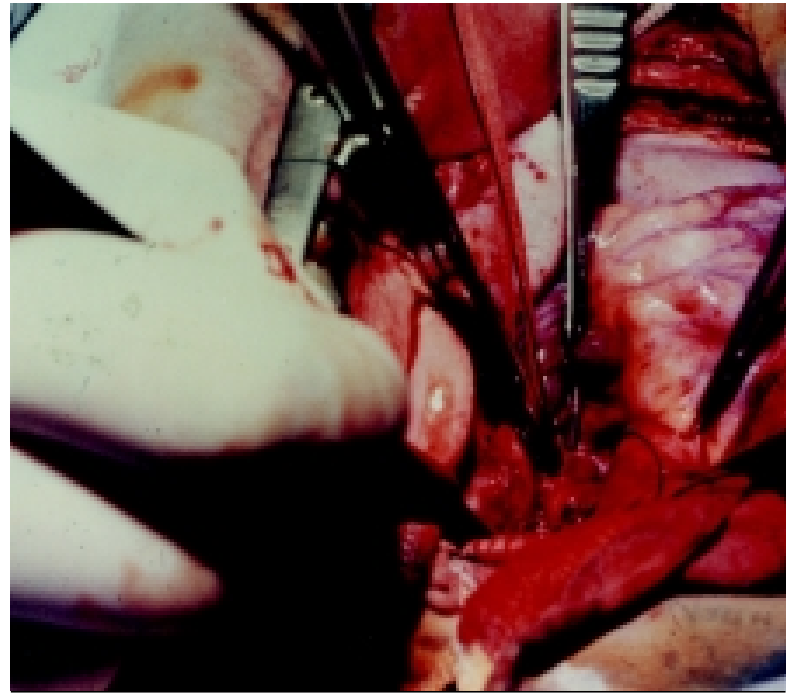

Fig. 3 - Detalhe da técnica operatória. Vaso anômalo oriundo de ramo direito da artéria pulmonar dirigindo-se ao AE posteriormente.

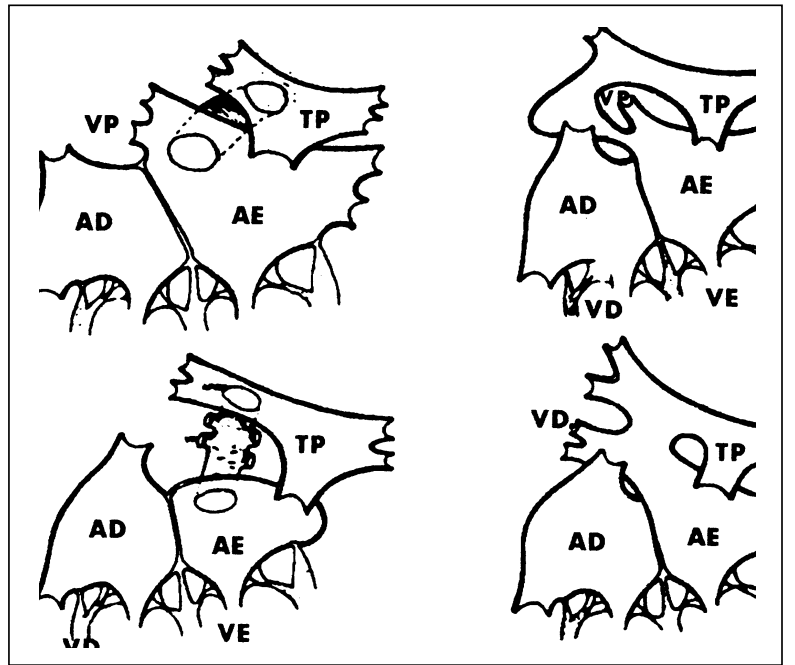

Fig. 4 - Tipos anatômicos de comunicação direta entre o ramo direito da artéria pulmonar e o átrio esquerdo. Tipo I (Sup. Esq.) Tipo II (Sup. D.) Tipo III (Inf. Esq.) Tipo IV (Inf. D.) segundo Ohara

associado a drenagem pulmonar anômala. O conhecimento desta morfologia é extremamente importante na programação cirúrgica adequada, pois um diagnóstico incorreto poderá levar à ligadura ou secção da comunicação em um ponto que excluiria o retorno venoso pulmonar ou mesmo a ligadura de um ramo da AP.

A primeira correção cirúrgica com sucesso foi realizado por Blalock, em 1948, relatado por AYTAC \& TUNCALI (11). A técnica cirúrgica utilizada desde então variou desde dissecção e ligadura simples, procedimento mais usado, até reparo intracardíaco em CEC, situação indicada quando o vaso anômalo e aneurismático ${ }^{(9)}$.

A literatura mostra uma sobrevida de mais de $70 \%$ e os óbitos sem tratamento cirúrgico são decorrentes de embolia ou abscesso cerebral (14).

Podemos afirmar que a comunicação direta entre APD e AE, apesar de cardiopatia congênita rara, é facilmente diagnosticada com angiografia e, atualmente, com ressonância nuclear magnética. A intervenção cirúrgica precoce é recomendável com mortalidade aceitável. Os pacientes não diagnosticados ou mantidos em observação clínica podem morrer em decorrência de ruptura da fístula, embolias ou abscesso cerebral. 
Schimin L C, Franceschini I A, Costa J N L, Lucena V S - Comunicação direta entre artéria pulmonar direita e átrio esquerdo: relato de dois casos. Rev Bras Cir Cardiovasc 2000; 15(4): 349-52.

RBCCV 44205-522

Shimin L C, Franceschini I A, Costa J N L, Lucena V S - Direct communication between the right pulmonary artery and the left atrium: two cases report. Rev Bras Cir Cardiovasc 2000; 15(4): 349-52.

ABSTRACT: The right pulmonary artery and left atrium direct communication is a rare anomaly. There are no more than 40 cases reported worldwide. Clinical presentation is variable, and patients can exhibit cyanosis, respiratory distress or heart failure. However, diagnosis can be easily made by image tests. Surgical treatment offers total healing with a few complications and mortality. Clinical follow-up without surgery can lead to remarkable complications such as brain abscess. Two cases on direct communication between right pulmonary artery and left atrium are reported. The embryogenic theories and anatomic varieties of this congenital abnormality will be discussed.

DESCRIPTORS: Cardiac surgical procedures, methods. Heart atrium, surgery. Pulmonary artery, surgery.

\section{REFERÊNCIAS BIBLIOGRÁFICAS}

1 Sassolas F, Long $\mathrm{J}$ L, Bozio A et al. - Communication artère pulmonaire droite-oreillette gauche: une rare cause d'hipoxemie refractaire chez le nouveau-né. Pediatrie 1984; 39: 203-8.

2 Stuckey S - Direct communication between the right pulmonary artery and the left atrium: magnetic resonance findings. Australas Radiol 1993; 37: 216-20.

3 Almeida O D, Cortelazzi P S, Godoy M F et al. - Comunicação direta entre o ramo direito da artéria pulmonar e o átrio esquerdo: relato de um caso e revisão da literatura. Arq Bras Cardiol 1985; 45: 271-5.

4 Arendrup H - Direct communication between the pulmonary artery and left atrium. Scand $J$ Thorac Cardiovasc Surg 1982; 16: 157-60.

5 Lekuona I, Cabrera A, Inguanzo R, Cid C, Agosti J Direct communication between the right pulmonary artery and the left atrium. Thorax 1986; 41: 78-9.

6 Ito $\mathrm{T}$, Yokota $\mathrm{H}$, Murata $\mathrm{H}$ et al. - Direct communication between the pulmonary artery and left atrium: a case report and review of the literature. J Cardiovasc Surg 1986; 27: 108-12.

7 Kroeker E, Adams H, Leon A et al. - Congenital communication between a pulmonary artery and left atrium. Am J Med 1963; 34: 721-5.
8 Verel D, Grainger R, Taylor D - Direct communication of a pulmonary artery with the left atrium. Br Heart $J$ 1964; 26: 856-8.

9 Abe T, Kuribayashi R, Sato M, Nieda S - Direct communication of the right pulmonary artery with the left atrium: a case report and review of literature. $J$ Thorac Cardiovasc Surg 1972; 64: 38-44.

10 Lucas R V, Lund G W, Edwards J E - Direct communication of a pulmonary artery with the left atrium: an unusual variant of pulmonary arteriovenous fistula. Circulation 1961; 24: 1409-14.

11 Aytac A \& Tuncali T - Direct communication of a pulmonary artery with the left atrium: first reported case of all four pulmonary veins entering the communication. $J$ Thorac Cardiovasc Surg 1967; 54: 553-6.

12 de Souza e Silva N A, Giuliani E R, Ritter D G, Davis G $\mathrm{D}$, Pluth J R - Communication between right pulmonary artery and left atrium. Am J Cardiol 1974; 34: 857-63.

13 Ohara $\mathrm{H}$, Ito $\mathrm{K}$, Kohguchi $\mathrm{N}$ et al. - Direct communication between the right pulmonary artery and the left atrium: a case report and review of the literature. J Thorac Cardiovasc Surg 1979; 77: 742-7.

14 Miao W Z, Qian J Q, Chen X Y, Shao S C, Wang S, Chen $E$ - Direct communication between right pulmonary artery and left atrium: a case report. Chin Med $J$ (Engl) 1984; 97: 623-6. 